\title{
TEORÍAS LINGÜÍSTICAS DEL HUMOR VERBAL
}

\author{
Torres Sánchez, María Ángeles \\ Universidad de Cádiz, Departamento de Filologia, Area de Linguística, \\ Facultad de Filosofia y Letras, Bartolomé Llompart s/n, 11003 Cádiz. \\ Tfno. (956) 245009, Fax. (956) 220444.
}

(Recibido Julio 1997, aceptado Diciembre 1997)

BIBLID [1]33-682X (1997-1998) 5-6, 435-448.]

\author{
ATTARDO, S. (1994), Linguistics theories of humor, Berlin \& New York, Mouton \\ de Gruyter, Pp. xix, 426.
}

\section{Resumen}

En este artículo se ofrece una revisión crítica de la obra Linguistics theories of humor (Attardo, 1994), que constituye uno de las pocos trabajos básicos para el estudio del humor verbal desde una perspectiva lungüistica. Se presenta una revisión general de los numerosos estudios llevados a cabo sobre el humor desde perspectıvas distintas (antropológica, literaria, filosófica, psicológica, socıológica o folklórıca), con el objetivo de traducir las conclusiones de dichos trabajos en términos lingúlsticos, y aplicar diversos modelos linguisticos al análisis del humor verbal, para concluir con una propuesta de analisis desde la línea pragmática, que nosotros adoptamos como la más adecuada para la interpretación del fenómeno humoristico en la comunicación.

Palabras clave: pragmática, humor verbal, puns, chistes.

\begin{abstract}
This article presents a critical examination of Attardo's (1994) Lingustics Theores of Humour This book Is one of the few basic studies about verbal humour from a linguistic perspective. A general overview is offered of the numerous studies about humour from different perspectives (anthropological, literary, philosophical, psychological, sociological, etc.), with the purpose of interpreting their conclusions in linguistic terms, and applying different linguistic models to the analysis of verbal humour. This paper concludes proposing an analysis of verbal humour from a pragmatic perspective, which, in our opınion, is the most appropriate line of research for the study of humorous interpretation.
\end{abstract}

Key words: pragmatics, verbal humour, puns, jokes.

Pragmalingüistica 5-6, 1997-1998, 435-448. 
Torres Sánchez, M" Ángeles - Teorias linguisticas del humor verbal

\section{Résumé}

Dans cet article ou offre une révision critique de l'ouvrage Lingusstic Theores of Humor, que est un de peu abondants travaux basiques pour l'étude de l'humour verbale dés une perspectıve linguistıque. Ou présente une révisjon générale de nombreuses études réalisées sur l'humour dès des perspectives différentes (anthropologıque, littéraire, philosophique, psicologique, sociologique ou folclorıque) à l'object de traduır les conclusions des travaux mentionés en termes linguistrques, et appliquer de distincts modèles linguistiques à l'analyse dès la ligne pragmatique, que nous adoptons comme la plus appropiée pour l'interprétation du phénomène humouristique dans la communication.

Mots-clés' pragmatique, humour verbale, puns', blagues.

Linguistics theories of humor constituye una de las pocas obras que se pueden considerar como básicas para el estudio del humor verbal desde una perspectiva lingüística. Este libro ofrece una revisión general de los numerosos trabajos sobre el humor realizados desde perspectivas distintas (antropológica, literaria, filosófica, psicológica, sociológica o folklórica), con el objetivo de traducir las conclusiones de dichos trabajos en términos lingüísticos, y aplicar diversos modelos linguísticos al análisis del humor verbal. Este doble objetivo confiere al libro de Attardo un carácter más descriptivo que explicativo, a pesar de que el autor intenta al mismo tiempo exponer algunas de sus ideas particulares sobre el humor a través del análisis de dos de las manifestaciones humorísticas más destacadas, a saber, los puns y los chistes.

Los términos humor y humorismo se han relacionado a lo largo de la tradición con otras nociones, como las de "juego verbal", "arte verbal", "cómico" o "divertido". Ya en la "Introducción" (Introduction, 1-13) Attardo establece una distinción básica entre lo que antropológicamente se considera "émico", por ocasionar la risa o el aplauso de los individuos, y lo que los teóricos consideran divertido, lúdico o artístico en función de la intención creadora $y$ las características estructurales que marcan estas manifestaciones lingüísticas del humor. Attardo insiste en que la clave de todo humor se halla en la competencia, entendida ésta en el sentido que los linguistas que siguen la línea de Chomsky dan al término competencia, esto es, como "conocimiento tácito".

El capítulo primero, "Revisión de la literatura" (Survey of the literature, 14-59) muestra la amplitud tanto temporal como disciplinar del término "humor". Revisa el autor los estudios de los griegos (Platón, Aristóteles, etc.) y los romanos (Cicerón, Quintiliano, etc.), así como la escasez investigadora sobre el tema en la Edad Media, para llegar hasta el Renacimiento y la Modernidad, prestando especial atención a Bergson (1900) y Freud (1905, 1966), cuyos trabajos han de tenerse en cuenta por todo aquel que se interese por el humor, cualquiera que sea la perspectiva o disciplina adoptada. Un aspecto especialmente interesante de este capitulo es la clasificación de las

Pragmalinguística 5-6, 1997-1998, 435-448. 
teorías modernas sobre el humor elaborada por el autor, distribuidas éstas en tres grupos, que responden a los siguientes epígrafes:

a) Teorías de la superioridad, que defienden que toda experiencia humorística surge como manifestación del sentimiento de superioridad del hombre hacia el hombre.

b) Teorías de la descarga, que interpretan el humor como efecto de una descarga de energía física acurnulada.

c) Teorías de la incongruencia, que consideran que todo humor se basa en el descubrimiento de una realidad o un pensamiento que resulta incongruente con lo que se esperaba.

La parte central del libro, de esencial interés para los linguistas, está conformada por una serie de capítulos sobre los chistes y los "puns". Tal vez uno de los problemas más destacables en este bloque sea el de las definiciones y características de ambos fenómenos, que Attardo da por sabidas y no explicita ni discute. Sin embargo, es cierto que tanto los puns como los chistes piantean serias dificuitades de definición y análisis por diversas razones. En primer lugar, porque ni unos ni otros se distinguen claramente de otras formas lingüisticas: los puns se pueden considerar, en ocasiones, como casos de homónimos, metáforas o expresiones proverbiales; los chistes, por otro lado, pueden incluirse dentro del conjunto general de textos narrativos. En segundo lugar, porque pueden ser estudiados desde diferentes perspectivas y con diversos objetivos, y llegar así a resultados distintos e incluso contrarios. Por ejemplo, si los chistes se analizan como textos aislados de la conversación real, presentan un evidente carácter monológico; sin embargo, si los estudiamos en términos de actuación, incluyendo un foco interactivo y prestando especial atención a la manifestación de la risa ocasionada u otras posibles reacciones, han de considerarse, entonces, como inherentemente dialógicos. Los puns, por otro lado, pueden considerarse como conscientes o no conscientes, según el punto de vista adoptado, o tener un propósito o no tenerlo, dando así lugar a análisis e interpretaciones muy diferentes. Disentimos de Attardo cuando implica, y de hecho asume, que los puns y los chistes tienen un carácter universal, a no ser que nos situemos en un nivel general. Lo normal, en nuestra opinión, es que ambas manifestaciones humorísticas atraigan la atención del linguista siempre y cuando su estudio pueda arrojar alguna luz en el análisis de algún otro problema lingüístico concreto de una lengua determinada.

En el segundo capitulo, "La organización lineal del chiste" (The linear organization of the joke, 60-107) se presenta un modelo para el análisis estructural de los chistes, basado en los modelos de análisis narrativo desarrollados por Greimas. La estructura del chiste, según Attardo, es bastante simple, puesto que describe sólo las caracteristicas narrativas de los textos humorísticos:

Pragmalinguistsca 5-6, 1997-1998, 435-448. 
the text begins by setting a context... An element...then occurs in the text which causes a passage from the sense reconstructed thus far in the joke to a second, opposed sense. This element occurs at the end of the text, a position which is the location of the rheme of the last sentence of the text. (p. 107).

La estructura que aquí se ofrece es completamente monológica de base textual. A pesar de que esta descripción de la estructura del chiste es simple, la discusión que se presenta en el capitulo es muy compleja, y hace uso de la terminología de Greimas (1966)(isotopía, función narrativa, disyunción, etc.), tras ser discutida con cierto detalle. Si bien Greimas insiste en que las isotopías actúan en la superficie del texto, se ha reconocido que nociones como las de "coherencia" y "tópico" operan en un nivel distinto, y requieren la consideración de la inferencia y el conocimiento del mundo (cf. van Dijk, 1972, 1980; Reinhart, 1981). El concepto de isotopía de Greimas es un concepto semántico, mientras que el concepto de coherencia es pragmático. Esto indica que tanto la noción original como los intentos por refinarla ignoran distinciones cruciales. Obviamente, el estudio del significado discursivo no puede seguir las líneas sugeridas por Greimas. A pesar de las dificultades y de los evidentes problemas que plantea, este marco teórico se ha empleado repetidamente en el estudio del humor verbal. En este capítulo, Attardo también proporciona una tipología de los chistes, en la que distingue los chistes referenciales y los verbales, basados éstos últimos fundamentalmente en caracteristicas particulares de la realización fonológica en los mismos. No obstante, se podrian establecer muchas otras tipologías, a partir de las dimensiones estructurales, culturales y sociológicas que presentan los chistes.

Entre los trabajos que han revisado las características estructurales del chiste y el llamado "conector" en la teoria de Greimas, Charaudeau (1972) arguye que el término conector, que camufla la oposición de isotopías, y el disyuntor, que la revela, no se sobreponen, como defiende Greimas (Attardo 1994: 87). Bárdosi (1976), por su parte, aplica esta idea al análisis de los chistes en húngaro (Attardo 1994: 90).

Attardo también menciona el trabajo de Haussman (1974, citado en Attardo, 1994: 92), en el que se aplica el modelo de Greimas al estudio de los "puns", distinguiendo tres tipos de coherencia: lingüística, objetiva (establecida a través del conocimiento del mundo), y convencional (que surge de expresiones congeladas y frases hechas). Haussman considera el concepto de isotopía como idéntico al de coherencia lingüística, que, según él, queda rota en los "puns". Por ello, el discurso humorístico es caracterizado como "texto desviado", dado que la coherencia se considera como el rasgo fundamental para la unidad textual en los textos no humorísticos. Según el resumen que Attardo hace de este trabajo, está claro que Haussman no aplica realmente las ideas de 
Greimas en su análisis del humor. Es conveniente recordar que el objetivo final de Greimas consistía en demostrar que todo el significado está en el texto. El hecho de que Haussman hable del "conocimiento del mundo" es una indicación más de la imposibilidad de estudiar el humor verbal desde una perspectiva exclusivamente gramatical.

La mayoria de los trabajos que se han realizado bajo la influencia de Greimas se limita al análisis de los chistes, y se concentra en su estructura lineal. Los estudios dentro de este marco coinciden en la existencia de un disyuntor, que provoca un paso del sentido reconstruido hasta ese momento en el chiste a un segundo sentido, opuesto al primero como rasgo crucial en la estructura del chiste. Los análisis cuantitativos de un gran número de chistes han demostrado que los disyuntores aparecen siempre al final del chiste, en la posición del rema de la última oración del texto', de manera que se puede prescindir de cualquier material lingüistico que aparezca detrás de él sin que se produzca ningún efecto importante en el proceso interpretativo del chiste. Este hecho se puede interpretar como indicador de su "no-rematicidad". Esta "no-rematicidad" del material que sigue al disyuntor es, según Attardo, específica de la estructura del chiste (Attardo, 1994: 101) ${ }^{2}$.

Por su propia naturaleza, estos acercamientos semióticos, que desde el punto de vista comunicativo se basan en lo que Sperber y Wilson (1986) denominan el Modelo del Código y sólo consideran los aspectos lingüísticamente codificados del mismo, no dan cuenta, pues, del proceso de interpretación humorística como algo perteneciente al campo de la pragmática. Sin embargo, el hecho de que el humor verbal sea un fenómeno esencialmente psicológico nos plantea la cuestión de cuáles son los procesos que provocan esa experiencia psicológica. Los estructuralistas y los semióticos dan por sentada una correlación causal entre la existencia de distintos tipos de textos humorísticos y nuestra reacción hacia ellos, y no consideran el estudio de esta conexión como un problema para las teorías de la comunicación, y por lo tanto, restringen el estudio del humor verbal a la caracterización de las propiedades estructurales de los

\footnotetext{
1 Las nociones tema y rema son categorias formales, empleadas por los analistas del discurso, para distinguir entre información dada e información nueva, respectivamente. Para una discusión más detallada de los procesos de tematización, cf. Halliday (1967), Brown y Yule (1983) y Halliday y Hassan (1985)

2 Attardo presenta el siguiente ejemplo para ilustrar esa idea:

"Do you believe in clubs for yound people?", "Only when kindness fails, my friend", que también podría terminar así "Only when kindness fails, replied Fields". El disyuntor aquí, según Attardo, es "when kindness fails". Todo lo que le sigue (en este caso "replied Fields") puede elıminarse sin ningún efecto en el chiste (Attardo, 1994: 99).
}

Pragmalınguistıca 5-6, 1997-1998, 435-448 
textos humorísticos. Esta crítica a las versiones más estrictas dentro del Modelo del Código, se puede extender igualmente a otros trabajos más orientados hacia un modelo inferencial de interpretación comunicativa. En esta misma línea, Attardo, desarrollada un análisis del humor desde una perspectiva inferencial, siguiendo la teoría de Grice (1975):

A theory of the processing of the text of jokes must distinguish two moments in the disambiguation of a joke text (for the sake of simplicity, "joke" will be used hereafter instead of "joke text"): in the first part of the process, a first isotopy/sense $\left(S_{1}\right)$ is established, until a recipient encounters an element that causes the passage from the first sense to a second sense $\left(S_{2}\right)$ antagonistic to the first one (...) The passage from $S_{1}$ to $S_{2}$ must be "unexpected", on the one hand, and "immediate" (i.e., should not involve "exceptional" mental expenditure), on the other. (Attardo, 1994: 95).

Sin embargo, el autor no considera la "teoría del procesamiento de los chistes" como objeto de estudio de la lingüística sino de la psicología, como deja claro en una nota a pie de página:

Although these notions are rather vague, they are widely accepted in humour theory, and their definition is a problem for psychology, not linguistics. (Attardo, 1994: 95)

La psicología, sin embargo, no ha asumido este reto, y son escasos los trabajos que se han planteado explicar en profundidad cómo se procesan los textos humorísticos. Por último, de lo que Attardo denomina "Teorías lingüisticas del humor", se desprende que el autor se refiere exclusivamente a la lingüística del texto.

Los capitulos 3, "El análisis de los puns" (The analysis of puns, 108-142) y 4, "La resolución de los puns" (Resolution in puns, 143-173) examinan el fenómeno humoristico de los "puns". Según Attardo (108), los "puns" se producen conscientemente, como los chistes hablados, escritos o firmados. El autor señala que en la lingüística estructural se han establecido taxonomías de la formación de "puns" (y presenta varias de estas taxonomías) y se ha documentado la existencia de "puns" en lenguas no indoeuropeas. También revisa el autor ciertas investigaciones recientes sobre la relación entre los "puns" y la distancia fonémica. Es interesante notar, como hace Attardo, que los "puns" se han empleado menos como material de análisis lingüístico que otros fenómenos del uso del lenguaje como la poesía, el análisis de errores, y los juegos

Pragmalinguistica 5-6, 1997-1998, 435-448. 
lingüísticos.

El capítulo quinto, "La semiótica y las teorias del texto" (Semiotic and text theories, 174-194) presenta una serie de modelos para el análisis del humor que coinciden en el estudio de los fenómenos humorísticos con arreglo al contexto semiótico o textual en el que se presentan. Se incluyen los trabajos de Eco (1976), Koestler (1964), Milner (1972), Dorfles (1968) y Manetti (1976), entre otros. Estos autores intentan ir más allá de la estructura del chiste, para dar cuenta de textos mayores y de las características no verbales en ellos. Estos nuevos estudios sobre el humor tienden a ceñirse a la noción de "oposición" presentada por Greimas, pero abandonan la idea de que el humor verbal derive sólo de los aspectos del texto lingüísticamente codificados. La idea de que la oposición sea central en la generación de un efecto humorístico, sin embargo, no es exclusiva de Greimas. Muchos trabajos convergen en una cierta "bisociación", definida por Koestler (1964: 35) en los siguientes términos:

...the perceiving of a situation or idea... in two self-consistent but habitually incompatible frames of reference... The event... in which the two intersect, is made to vibrate simultaneously on two different wavelengths, as it were. While this unusual situation lasts, (the event) is not merely linked to one associative context, but bisociated with the two.

En la tradición semiótica, Milner (1972: 16) señala:

within a single situation, and a single linguistic context, two unverse collide, and it is this collision that makes many forms of humor possible.

Milner define el "pun" como "la inversión paradigmática de dos ítemes", y apunta cinco niveles en los que se produce esta inversión: el fonológico, el morfológico, el sintáctico, el léxico y el situacional.

Los semióticos han considerado frecuentemente el humor como un proceso de ajenación de un signo que tiene lugar cuando se extrae de su contexto más estereotípico. Cuando esto ocurre, el signo ya no se remite a su "referente natural", sino a otro referente "paradójico", y, por ello, adquiere un valor negativo. En consecuencia, el humor se considera como un tipo de lenguaje... caracterizado por el supuesto valor negativo o paradójico del signo. (Dorfles, 1968: 104).

Manetti (1976), basándose en la hipótesis de la ajenación de Dorfles, propone seis mecanismos de ajenación (la metonimia, la metáfora, cambios en el sujeto de la enunciación, decontextualización, paralelismos y deformación), y también desarrolla la idea de que el discurso humorístico es una desviación a través del análisis de las palabras

Pragmalingüisstca 5-6, 1997-1998, 435-448 
que constituyen la clave humorística del chiste, en términos de la teoría de la información. Según Manetti estas palabras están muy marcadas informativamente ${ }^{3}$. Para apoyar el argumento según el cual los textos humorísticos constituyen una desviación de la norma, Manetti se basa en la doble isotopía de Greimas. El discurso serio es unívoco, y generalmente ambiguo, y responde a una sola isotopia. El discurso humoristico, por el contrario, opone sistemáticamente dos isotopías. En función del tipo de contraste isotópico, Manetti propone una clasificación de los posibles tipos de humor.

Esta breve revisión demuestra que la perspectiva semiótica considera el humor verbal no como una propiedad de los procesos de interpretación, sino como característica de la relación entre los signos y sus contextos. Por otra parte, tanto las interpretaciones semióticas como las textuales, se aferran a la idea de que el discurso humorístico es un discurso marcado o desviado, en oposición al discurso serio. Attardo nota, y de hecho critica, que la mayor parte de estos trabajos son excesivamente programáticos, incompletos y poco detallados, y tienden a basar sus teorías en datos parciales obtenidos de un número limitado de ejemplos.

En el capítulo sexto, "Las teorias basadas en guiones" (Script-based theories, 195-229), Attardo trata la Teoría de los Guiones de Raskin (1981), como otra posible vía de interpretación del humor inspirada en la gramática generativa. La aportación básica de este trabajo consiste en la explicación de las intuiciones/competencia/habilidades de los hablantes nativos para reconocer si un texto es jocoso o no. Attardo discute si tal extensión del concepto de competencia lingüística es legítimo, y defiende que si lo es. En realidad, este argumento debe ser parte de una discusión más amplia ‘la relación entre la competencia gramátical de Chomsky y la supuesta competencia pragmática y sociolingüistica, que ha sido el centro de atención de los estudios de Hymes (1967) y otros autores.

Los capítulos 7, "El humor basado en el registro" (Register-based humor, 230253), y 8 "Textos humorísticos que no son chistes" (Non-joke humor texts, 254-270), constituyen una exploración más profunda de las teorías del humor más allá del chiste. El capitulo 7 trata el estilo (situacional y social) y el humor, y por ello incluye algunos aspectos de las relaciones entre la sociolingüística y el humor. Attardo no se detiene demasiado en este punto, a pesar de que ésta podría ser una área de investigación fructífera. En el capítulo 8 Attardo ilustra la teoría del humor basada en los guiones examinando cuatro textos, una historia breve de Alla Poe, un fragmento de Cándida de Voltaire, otros extraídos de las novelas de Thomas Love Peacock, y un pasaje de un texto medieval francés. Como señala Attardo, este ejercicio presenta bastantes elementos

${ }^{3}$ Esta línea de investigación también se sigue fuera de la tradición semiótica. Cf., por ejemplo, Giora (1991).

Pragmalinguistica 5-6, 1997-1998, 435-448 
en común con la crítica literaria de textos.

Ya en el capítulo noveno, "La naturaleza cooperativa del humor" (The cooperative nature of humor, 271-292), el autor analiza los chistes y el humor en general desde la perspectiva del Principio de Cooperación y las máximas conversacionales de Grice, y apunta que mientras que los chistes no son cooperativos en el sentido griceano, y suponen una violación intencionada de las máximas, claramente expresan un mensaje; por ello, son un aspecto de la competencia comunicativa y deberían ser incorporados en una teoría más amplia de tal competencia, gobemada por un conjunto de máximas diferentes a las propuestas por Grice (1975). Éste último consideraba no sólo la ironía, sino también la metáfora, la meiosis y la hipérbole como casos de violación de la primera máxima de cualidad ("no diga Vd. aquello que crea falso"). En aquellas circunstancias en las que resulta obvio, tanto para el hablante como para el oyente, que el hablante no cree en la veracidad de sus palabras, surge la inferencia de que éste debe de estar tratando de comunicar un proposición relacionada con aquélla que ha expresado, pero distinta. Grice defiende que en casos de ironía "la proposición más obviamente relacionada con aquella que el hablante expresa es justamente aquélla que la contradice" (Grice 1989: 34); esta proposición se recupera a través de la noción de implicatura. Si bien la ironía no es siempre humorística, lo cierto es que siempre constituye un caso de ingenio verbal.

Attardo arguye que los siguientes ejemplos ilustran cómo puede surgir un efecto humorístico de la violación de cada una de las máximas (Attardo, 1994: 272):

\section{(1) Cantidad.}

A: Excuse me, do you know what time it is?

Bi $\quad$ Yes.

(2) Relación.

A: "How many surrealists does it take to screw in a light bulb?"

B: "Fish".

(3) Modo.

A: "Do you believe in clubs for young people?"

B: "Only when kindness fails": 


\section{Cualidad.}

A: Why did the Vice President fly to Panama?

B: Because the fighting is over.

Attardo defiende que to que hace que estos casos sean especiales es que no respetan las máximas conversacionales, sino que las violan, y señala que ninguna derivación de las implicaturas restaurará el supuesto de que se está respetando el principio de cooperación. Lo que se viola, entonces, es el propio principio de cooperación, de manera que, en términos de Grice, el hablante está "opting out". En opinión de Attardo (1994: 275), esto plantea el siguiente problema: ¿cómo es posible, entonces, que los hablantes se comuniquen con éxito en intercambios de carácter humorístico? El problema surge, según Attardo, porque a pesar de que estos ejemplos son casos de comportamiento no cooperativo, sin embargo tienen sentido, y se interpretan y se reconocen como chistes.

Esto, no obstante, plantea dificultades; considérese el ejemplo (1). Si bien es cierto que puede resultar divertido en determinadas circunstancias, también puede resultar grosero, o simplemente no cooperativo. aunque, a nuestro juicio, en un sentido distinto a la falta de cooperatividad que lleva al oyente a cambiar al tipo de comunicación de "no serio" (non-bona-fide)(según las características que Attardo y Raskin (1991) dan del humor. ¿De qué mecanismos dispone el oyente para interpretar este ejemplo como indice de que el hablante quiere ser rudo o como un comentario humorístico? El hecho de que estas dos interpretaciones alternativas resulten difíciles de discriminar es algo de lo que una teoría del humor debe dar cuenta. De hecho, algunos intentos de humor fracasan precisamente en este punto, $y$ en ocasiones provocan una ofensa al oyente. Los modelos de Raskin y Attardo, sin embargo, no consideran esta distinción.

Tanto Attardo como Raskin proponen una explicación a esta "paradoja". Raskin (1985) sugiere no sólo una teoría general de la comunicación, sino también un principio de cooperación diferente que se encarga de guiar los intercambios que se producen en el modo de comunicación no serio. Los dos principios de cooperación no entran en conflicto porque también se establece una jerarquía entre ambos principios (Raskin, 1992; Attardo, 1994). En el nivel inferior entra en juego el principio de cooperación griceano, mientras que el principio de cooperación del humor puede respetar el principio de cooperación original, aunque también permite violaciones de éste último siempre y cuando éstas se vean posteriormente compensadas por una segunda intención humorística (Attardo, 1994: 287). Asi, en el modo de comunicación no serio (non-bona-fide) la violación de una máxima no responde a la intención de seguir otra máxima distinta, sino 
que se viola para seguir un principio de cooperación de distinta naturaleza. Aparentemente la "intención humorística" es una inferencia por defecto. Raskin se refiere, por lo que se desprende de su análisis, a un procesamiento en dos etapas, por el que las máximas sólo entran en escena para explicar el hecho de que el narrador, después de todo, no está siendo cooperativo. Sin embargo, nos planteamos la siguiente cuestión: ¿necesitamos comprobar posteriormente que el narrador ha seguido tales máximas para llegar a la conclusión de que estaba contando un chiste? Obviamente no, dado que, como arguye Yamagushi (1988), la violación es sólo temporal, y al proporcionar después una información anteriormente omitida, demuestra que ésta estaba siendo retenida con la simple intención de generar la ambigüedad en la que se basa el efecto humorístico. Esto es suficiente por sí mismo para mantener en todo momento la existencia de una presunción de cooperatividad. De hecho, cuando los hablantes son realmente no-cooperativos, como al mentir o al ocultar información a los hablantes, no proporcionan evidencia inmediata de que se ha violado el principio de cooperación o no corrigen su transgresión de las máximas, como es el caso precisamente de los chistes.

El capitulo 10, "El humor en contexto" (Humor in context, 293-331), versa sobre distintos aspectos de la relación entre los chistes y los "puns" y los contextos en los que ocurren, principalmente desde la perspectiva del análisis conversacional. En este capítulo, Attardo reconoce la posibilidad de "puns" inconscientes y inconscientes en la conversación. Finaliza el capítulo con una discusión sobre las funciones comunicativas y sociales del humor.

El capitulo 11, "Direcciones en la investigación sobre el humor" (Directions in humor research, 332-334), es una breve sugerencia del autor acerca de las futuras lineas de investigación posibles por las que se podría seguir avanzando en el estudio del humor verbal. Aquí, como en otros momentos del libro, Attardo lamenta la falta de comunicación entre los estudiosos en las distintas disciplinas, incluida la lingüística, que potencialmente pueden aportar contribuciones significativas al estudio del humor. Esta situación puede remediarse, dada la continua expansión de la lingüística en áreas tan relacionadas como el análisis de la conversación, el análisis del discurso, la etnografia del habla y la pragmática.

Sigue a este último capitulo una amplia y útil bibliografía, seguida de dos apéndices, en los que se proporciona numerosos ejemplos textuales del humor.

La relación, claramente fascinante, entre el lenguaje y el humor requiere futuras investigaciones, ya sea siguiendo la línea de Attardo, u otras posibles líneas de investigación. En nuestra opinión, una teoría satisfactoria del humor verbal, debe considerar este tipo de comunicación como un fenómeno que integra la estructura lingüistica, el juego en el habla y el arte verbal, dentro de los contextos sociales y culturales del uso del lenguaje. Esta consideración del humor como juego comunicativo 
Torres Sánchez, $M^{a}$ Angeles - Teorias linguisticas del humor verbal.

ha de recibir, por tanto, un análisis de carácter pragmático, al ser esta perspectiva la más adecuada desde nuestro punto de vista para la explicación integral del fenómeno humorístico. 


\section{Referencias}

ATTARDO, S. (1994), Linguistic Theories of Humour, Berlin, Mouton de Gruyter.

ATTARDO, S. y RASKIN, V. (1991), "Script theory revis(it)ed: joke similarity and joke representation model.", Humor, 4-3/4, 293-347.

BARDOSI, V. (1976), "Polysémie et homonimie comme sources linguistiques du comique.", Annales universitatis scientiarum budapestensis du Rolando Eotvós nominatae. Sectio linguistica, 7, 51-67.

BERGSON, H. (1900), Laughter, New York, Macmillan. (versión española, La risa. Ensayo de significación de lo cómico, Barcelona, Edic. Orbes S.A. (1986)).

BROWN, G. y G. YULE (1983), Discourse analysis, Cambridge, Cambridge University Press.

CHARAUDEAU, P. (1972), "Quelques procedés linguistiques de l'humour", Langues Modernes, 3, 63-73.

DIJK, T. van (1972), "La pragmática de la comunicación literaria", en Mayoral, J. A. (comp.) (1987), Pragmática de la comunicación literaria, Madrid, Arco/Libros S.A. 171-194.

DIJK, T. van (1980a), Estructuras y funciones del discurso, Madrid, Siglo XXI.

DIJK, T. van (1980b), Texto y contexto: Semántica y Pragmática del discurso, Madrid, Cátedra.

DORFLES, G. (1968), Artificio e natura, Turin, Einaudi.

ECO, H. (1976), A Theory of Semiotics, Bloomington, Illinois, Indiana University Press.

FREUD, S. (1905), " Der Humor", en Studienausgabe, vol.4, München.

FREUD, S. (1966), El chiste y su relación con lo inconsciente, Madrid, Alianza Editorial.

GIORA, R. (1991), "On the cognitive aspects of the joke", Journal of Pragmatics, 16, 465-485.

GREIMAS, J. (1966), Sémantique structurale, Paris, Larousse.

GRICE, H.P. (1975), "Logic and conversation", en Cole, P. y J.L. Morgan (eds.), Syntax and Semantics: vol. 3. Speech Acts, New York, New York Academic Press, 41 58.

GRICE, H.P. (1989), Studies in the Way of Words, Cambridge, Mass., Harvard University Press.

HALLIDAY, M. (1967), "Notes on transitivity and theme in English: Part 2.", Journal of Linguistics, 3, 199-244.

HALLIDAY, M. y R. HASSAN (1985), Language, context and text: aspects of language in a social-semiotic perspective, Oxford, Oxford University Press. 
HAUSSMAN, F. (1974), Studien zu einer Linguistik des Wortspiels. Das Wortspiel in Canard Enchainé, Tübingen, Niemeyer.

HYMES,D. (1967), "Models of the Interaction of language and social settings", Journal of Social Issues, 23 (2), 8-28.

KOESTLER, A. (1964), The art of creation, London, Hutchinson.

MANETTI, G. (1976), "Per una semiotica del comico", Il Verri 3, 130-152.

MILNER, G. (1972), "Homo ridens: toward a semiotic theory of humor and laughter". Semiotica, 5, 1-30.

RASKIN, V. (1981), "Script-based lexicon", Quaderni di Semantica, vol. II, 1, 25-34.

RASKIN, V. (1985), Semantic Mechanisms of Humor, Dordrecht, Reidel.

REINHART, T. (1981), "Pragmatics and linguistics: an analysis of sentence topics", Philosophica, 27, 1, 53-94.

SPERBER, D. Y D. WILSON (1986), Relevance. Communication and cognition, Oxford, Blackwell (segunda edición, 1995). (Trad. esp. E. Leonetti, La Relevancia, Madrid, Visor.

YAMAGUSHI, H. (1988), "How to pull strings with words. Deceptive violations in the garden-path joke", Journal of Pragmatics 12, 323-337.

Pragmalinguistica 5-6, 1997-1998, 435-448. 